\title{
Ideal spatial radiotherapy dose distributions subject to positional uncertainties
}

\author{
Mustafa Y Sir ${ }^{1}$, Stephen M Pollock ${ }^{1}$, Marina A Epelman ${ }^{1}$, Kwok L Lam ${ }^{2}$ \\ and Randall K Ten Haken ${ }^{2}$ \\ ${ }^{1}$ Department of Industrial and Operations Engineering, The University of Michigan, Ann Arbor, \\ MI 48109, USA \\ ${ }^{2}$ Department of Radiation Oncology, The University of Michigan, Ann Arbor, MI 48109, USA \\ E-mail: msir@umich.edu
}

Received 12 June 2006, in final form 27 September 2006

Published 24 November 2006

Online at stacks.iop.org/PMB/51/6329

\begin{abstract}
In radiotherapy a common method used to compensate for patient setup error and organ motion is to enlarge the clinical target volume (CTV) by a 'margin' to produce a 'planning target volume' (PTV). Using weighted power loss functions as a measure of performance for a treatment plan, a simple method can be developed to calculate the ideal spatial dose distribution (one that minimizes expected loss) when there is uncertainty. The spatial dose distribution is assumed to be invariant to the displacement of the internal structures and the whole patient. The results provide qualitative insights into the suitability of using a margin at all, and (if one is to be used) how to select a 'good' margin size. The common practice of raising the power parameters in the treatment loss function, in order to enforce target dose requirements, is shown to be potentially counter-productive. These results offer insights into desirable dose distributions and could be used, in conjunction with well-established inverse radiotherapy planning techniques, to produce dose distributions that are robust against uncertainties.
\end{abstract}

(Some figures in this article are in colour only in the electronic version)

\section{Introduction}

Three-dimensional conformal radiotherapy (3DCRT) and intensity-modulated radiotherapy (IMRT) allow a significant amount of control over the shape of the radiation dose distribution. As a consequence, tumour target volumes with complex irregular shapes can be treated with high doses of radiation while surrounding healthy tissues and organs at risk (OAR) are spared as much as physically possible (Shepard et al 1999). However, if not properly taken into account during treatment planning, uncertainties caused by daily setup procedures at the beginning of each treatment fraction, as well as the inter- and intra-fraction motion of the internal organs, 
can lead to significant differences between the dose distribution calculated by a treatment plan and the actual dose distribution delivered to a patient (Bortfeld et al 2004, van Herk 2004, Lujan et al 1999).

In state-of-practice radiotherapy treatment planning, a common method used to account for these uncertainties is to enlarge the clinical target volume (CTV) by a 'margin' to produce a 'planning target volume' (PTV) (ICRU 1993). The width of the margin is typically set to be some number $k$ times the standard deviation of the patient setup error and/or the internal organ motion (van Herk et al 2000, van Herk 2004).

Three questions immediately come to mind:

(a) is this enlargement procedure a reasonable way to account for uncertainties due to patient setup error and internal organ motion;

(b) if so, what value of $k$ should be used;

(c) when an OAR is located in the neighbourhood of a CTV, so that the use of margins usually leads to a conflicting situation where the PTV overlaps with an OAR (Li and Xing 2000, Baum et al 2006), how can we determine the tradeoff between maximizing target coverage and minimizing OAR complications?

The answer to these questions requires the existence of some agreed-upon underlying measure of performance for a treatment plan. In some recent analyses (e.g., van Herk et al (2000), McKenzie et al (2000), Stroom et al (1999), Killoran et al (1997)), the performance measure is essentially the coverage probability, i.e., the probability that 'no part of the CTV accumulates a dose less than, for instance, 95\% of that prescribed'; however no mention is made of the potential damage to OARs and healthy tissue that might be incurred by any particular plan. Others (Shepard et al 1999, Li and Xing 2000, Unkelbach and Oelfke 2004, Rehbinder et al 2004) use a quadratic cost structure to capture both under-treatment of the CTV and damage to OARs and healthy tissue.

This paper presents a simple method for calculating the ideal spatial dose distribution: one that minimizes the expected loss when there is uncertainty. The focus is on constructing ideal spatial dose distributions which are robust against uncertainty; the problem of how to deliver such dose distributions once they have been constructed is not addressed.

Throughout this paper the assumption is made that the spatial dose distribution is invariant to the displacement of the internal structures and of the whole patient (Bortfeld et al 2004, Li and Xing 2000). That is, for a given set of beam configurations and fluence profiles, the resulting dose distribution is assumed to be fixed in space and not affected by small displacements of the patient anatomy. Put another way, the organs move within a stationary 'dose cloud' (Bortfeld et al 2004) without affecting the cloud. Our goal is to find such a stationary dose cloud which is optimal under uncertainty with respect to some treatment loss function. The invariance assumption, although not always realistic, makes clear the effect of various parameters on the overall delivered dose. It also allows associating a point in the treatment room with a loss, rather than associating a point in the patient with a loss. The result is a simple and intuitive method for calculating the optimal amount of dose that should be delivered to any point in the treatment room. The model also provides qualitative insights into the suitability of using a margin at all, and (if one is to be used) how to select a 'good' margin shape.

\section{The general model}

This section presents a general model of treatment of a single CTV in the neighbourhood of a single OAR (in section 3.3 the model is extended to include multiple CTVs and OARs). 


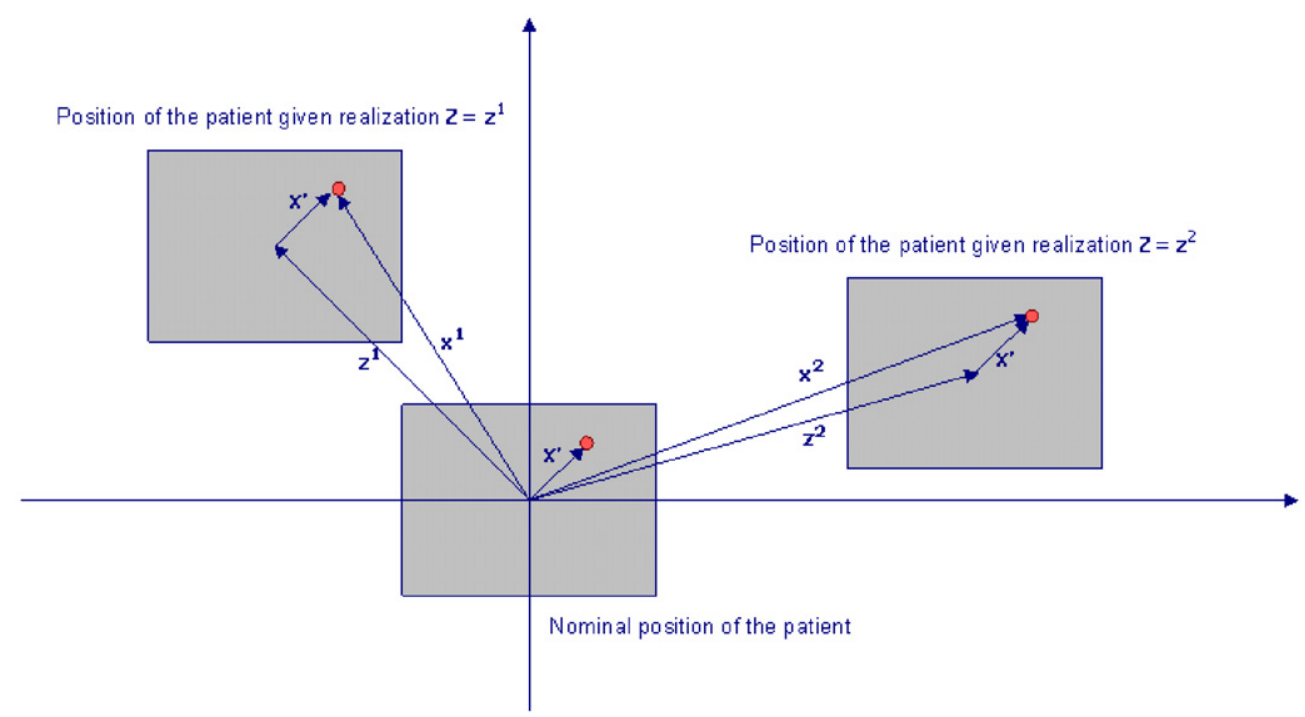

Figure 1. Illustration of random displacement. $\mathbf{x}^{\prime}$ is the room coordinate of the point with no displacement, i.e., the patient is in the 'nominal position'. $\mathbf{z}^{1}$ and $\mathbf{z}^{2}$ are examples of possible displacements. $\mathbf{x}^{1}$ and $\mathbf{x}^{2}$ are the room coordinates of the point given realizations $\mathbf{Z}=\mathbf{z}^{1}$ and $\mathbf{Z}=\mathbf{z}^{2}$, respectively.

The assumptions and notation are given first; later sections discuss specific forms of ideal dose distributions for certain critical functions. Throughout the paper, random variables are denoted by capital letters, their realizations by the respective lower case letters and vectors by bold face letters.

\subsection{General assumptions and definitions}

- $\mathbf{x}=\left[\begin{array}{l}x_{1} \\ x_{2} \\ x_{3}\end{array}\right]$ is a three-dimensional vector representing the coordinate of a point in the treatment room (relative to an arbitrary fixed origin). This coordinate system is referred to as the treatment room coordinate system, or simply room coordinates.

- $\mathbf{x}^{\prime}=\left[\begin{array}{l}x_{1}^{\prime} \\ x_{2}^{\prime} \\ x_{3}^{\prime}\end{array}\right]$ is the room coordinate of a point on the patient when there is no displacement.

- $\mathbf{Z}$ is a three-dimensional random vector representing the random displacement of the patient anatomy.

- The joint cumulative distribution function for $\mathbf{Z}$ is $F(\mathbf{z}) \equiv \operatorname{Prob} .\{\mathbf{Z} \leqslant \mathbf{z}\}=$ Prob. $\left\{Z_{1} \leqslant z_{1}, Z_{2} \leqslant z_{2}, Z_{3} \leqslant z_{3}\right\}$. Let $\mathbf{X}$ be a random vector representing the room coordinate of a point $\mathbf{x}^{\prime}$ on the patient. From the above definitions, $\mathbf{X}=\mathbf{x}^{\prime}+\mathbf{Z}$. Given realization $\mathbf{Z}=\mathbf{z}$, the point $\mathbf{x}^{\prime}=\mathbf{x}-\mathbf{z}$ on the patient is located at the room coordinate $\mathbf{x}$ (see figure 1).

- As implied by the above definition of displacement of the patient anatomy, only rigid body motions are considered; rotations and organ deformation are ignored.

- The location of the patient when there is no displacement (i.e., $\mathbf{z}=\left[\begin{array}{lll}0 & 0 & 0\end{array}\right]^{T}$ ) is referred to as the nominal position.

- $t\left(\mathbf{x}^{\prime}\right)$ is the desired ('target') dose to be delivered to patient location $\mathbf{x}^{\prime}$. 
- $L(d, t)$ is the loss ('cost,' or 'penalty') associated with delivering dose $d$ to a point having dose target $t$. An implicit assumption is made that the (biological) loss at any point does not depend on the dose at any other point. In other words, the loss associated with irradiation of a part of a structure is independent of irradiation of other parts of that structure. In effect, the loss function only captures local effects. Although this assumption is valid for many clinical situations and has been extensively used, there are some situations (for example, when organ effects are important) for which it is not valid (Alber and Nüsslin 1999).

- $y(\mathbf{x})$ is the dose delivered to the point on the patient located at room coordinate $\mathbf{x}$. The function $y(\cdot)$ represents the spatial dose distribution, assumed to be invariant to displacement of the internal structures and of the whole patient. In other words, it is the dose cloud within which the organs move without affecting the cloud (Li and Xing 2000, Bortfeld et al 2004). Since $y(\mathbf{x})$ is the result of decisions made by treatment planners, it is referred to as the 'planned' dose distribution. When $\mathbf{Z}=\mathbf{z}$, the dose delivered to patient location $\mathbf{x}^{\prime}$ is

$$
d\left(\mathbf{x}^{\prime}\right)=y\left(\mathbf{x}^{\prime}+\mathbf{z}\right) .
$$

Note that $y(\mathbf{x})$ is exactly what van Herk calls the 'desired/ideal dose distribution' $D_{\text {planned }}(x)$ (van Herk et al 2000). If there were no uncertainty, we would have liked to have $y(\mathbf{x})=t(\mathbf{x})$.

- $\mathcal{S}_{t}$ and $\mathcal{S}_{o}$ are three-dimensional regions on the patient representing CTV and OAR, respectively:

$$
\begin{aligned}
& \mathcal{S}_{t}=\left\{\mathbf{x}^{\prime}: \mathbf{x}^{\prime} \text { is a point in the CTV }\right\} \\
& \mathcal{S}_{o}=\left\{\mathbf{x}^{\prime}: \mathbf{x}^{\prime} \text { is a point in the OAR }\right\}
\end{aligned}
$$

- Given a point at room coordinate $\mathbf{x}, \mathcal{S}_{t}(\mathbf{x})$ and $\mathcal{S}_{o}(\mathbf{x})$ are the sets of all realizations $\mathbf{Z}=\mathbf{Z}$ such that the point is within the CTV and OAR, respectively:

$$
\begin{aligned}
& \mathcal{S}_{t}(\mathbf{x})=\left\{\mathbf{z}: \mathbf{z}=\mathbf{x}-\mathbf{x}^{\prime}, \mathbf{x}^{\prime} \in \mathcal{S}_{t}\right\}, \\
& \mathcal{S}_{o}(\mathbf{x})=\left\{\mathbf{z}: \mathbf{z}=\mathbf{x}-\mathbf{x}^{\prime}, \mathbf{x}^{\prime} \in \mathcal{S}_{o}\right\} .
\end{aligned}
$$

- $p_{o}(\mathbf{x})$ and $p_{t}(\mathbf{x})$ are the probabilities that a point at room location $\mathbf{x}$ will be covered by the OAR and CTV on the patient, respectively. That is,

$$
\begin{aligned}
& p_{o}(\mathbf{x})=\int_{\mathbf{z} \in \mathcal{S}_{o}(\mathbf{x})} \mathrm{d} F(\mathbf{z}), \\
& p_{t}(\mathbf{x})=\int_{\mathbf{z} \in \mathcal{S}_{t}(\mathbf{x})} \mathrm{d} F(\mathbf{z}) .
\end{aligned}
$$

\subsection{Calculating overall expected loss}

The overall expected loss is the performance measure used for evaluating any planned dose distribution $y(\cdot)$. In this subsection, formulae for calculating this measure are derived.

When the spatial dose distribution $y(\cdot)$ (which is invariant to displacement of the internal structures and of the whole patient) is used, the expected loss $\bar{L}_{P}\left[\mathbf{x}^{\prime}, y(\cdot)\right]$ at patient location $\mathbf{x}^{\prime}$ is given by the 'convolution' equation:

$$
\bar{L}_{P}\left[\mathbf{x}^{\prime}, y(\cdot)\right]=\int_{\mathbf{z}} L\left[d\left(\mathbf{x}^{\prime}\right), t\left(\mathbf{x}^{\prime}\right)\right] \mathrm{d} F(\mathbf{z})=\int_{\mathbf{z}} L\left[y\left(\mathbf{x}^{\prime}+\mathbf{z}\right), t\left(\mathbf{x}^{\prime}\right)\right] \mathrm{d} F(\mathbf{z}) .
$$


Equivalently, the expected loss $\bar{L}_{R}[\mathbf{x}, y(\cdot)]$ can be expressed in terms of room location $\mathbf{x}$ by substituting $\mathbf{x}^{\prime}=\mathbf{x}-\mathbf{z}$ in the right-hand integral of equation (8):

$$
\bar{L}_{R}[\mathbf{x}, y(\cdot)]=\int_{\mathbf{z}} L[y(\mathbf{x}), t(\mathbf{x}-\mathbf{z})] \mathrm{d} F(\mathbf{z}) .
$$

The total expected loss, $\Lambda[y(\cdot)]$, using spatial dose distribution $y(\cdot)$ is then:

$$
\Lambda[y(\cdot)]=\int_{\mathbf{x}^{\prime}} \bar{L}_{P}\left[\mathbf{x}^{\prime}, y(\cdot)\right] \mathrm{d} \mathbf{x}^{\prime}=\int_{\mathbf{x}} \bar{L}_{R}[\mathbf{x}, y(\cdot)] \mathrm{d} \mathbf{x} .
$$

Since, by assumption, the loss at any room location does not depend on the amount of dose delivered to any other location, the total expected loss $\Lambda[y(\cdot)]$ can be minimized by choosing the spatial dose distribution $y(\cdot)$ that minimizes the integrand at each point $\mathbf{x}$ of equation (9).

An 'optimal' dose distribution (or the ideal spatial dose distribution) is therefore given by the function $y^{*}(\cdot)$ that minimizes $\bar{L}_{R}[\mathbf{x}, y(\cdot)]$ for each value of $\mathbf{x}$. The resulting expected loss at patient location $\mathbf{x}^{\prime}$ is $\bar{L}_{P}^{*}\left(\mathbf{x}^{\prime}\right) \equiv \bar{L}_{P}\left[\mathbf{x}^{\prime}, y^{*}(\cdot)\right]$, the expected loss at room location $\mathbf{x}$ is $\bar{L}_{R}^{*}(\mathbf{x}) \equiv \bar{L}_{R}\left[\mathbf{x}, y^{*}(\cdot)\right]$ and the minimum total expected loss is

$$
\Lambda^{*}=\Lambda\left[y^{*}(\cdot)\right]=\int_{\mathbf{x}^{\prime}} \bar{L}_{P}^{*}\left(\mathbf{x}^{\prime}\right) \mathrm{d} \mathbf{x}^{\prime}=\int_{\mathbf{x}} \bar{L}_{R}^{*}(\mathbf{x}) \mathrm{d} \mathbf{x} .
$$

\subsection{Weighted power loss function}

An illustrative functional form for $L$, used in many IMRT treatment plan designs (see, for example, Tsien et al (2003), Kessler et al (2005) and Alber and Nüsslin (1999)), is the weighted power loss function (WPLF)

$$
L\left[d\left(\mathbf{x}^{\prime}\right), t\left(\mathbf{x}^{\prime}\right)\right]=w\left(\mathbf{x}^{\prime}\right)\left|t\left(\mathbf{x}^{\prime}\right)-d\left(\mathbf{x}^{\prime}\right)\right|^{b\left(\mathbf{x}^{\prime}\right)} .
$$

The weight $w(\cdot) \geqslant 0$ and power $b(\cdot) \geqslant 1$ are typically given by

$$
\begin{aligned}
& w\left(\mathbf{x}^{\prime}\right)= \begin{cases}w_{o} & \text { if } \mathbf{x}^{\prime} \in \mathcal{S}_{o} \\
w_{t} & \text { if } \mathbf{x}^{\prime} \in \mathcal{S}_{t}\end{cases} \\
& b\left(\mathbf{x}^{\prime}\right)= \begin{cases}b_{o} & \text { if } \mathbf{x}^{\prime} \in \mathcal{S}_{o} \\
b_{t} & \text { if } \mathbf{x}^{\prime} \in \mathcal{S}_{t} .\end{cases}
\end{aligned}
$$

Substituting (12) into (9), we obtain

$$
\bar{L}_{R}[\mathbf{x}, y(\cdot)]=\int_{\mathbf{z}} w(\mathbf{x}-\mathbf{z})|t(\mathbf{x}-\mathbf{z})-y(\mathbf{x})|^{b(\mathbf{x}-\mathbf{z})} \mathrm{d} F(\mathbf{z})
$$

when a WPLF is used.

\section{Calculation of optimal dose distribution}

Suppose the CTV has prescribed target dose $\tau\left(\mathbf{x}^{\prime}\right), \mathbf{x}^{\prime} \in \mathcal{S}_{t}$, while the OAR has target dose zero.

Recalling that $\mathbf{x}^{\prime}=\mathbf{x}-\mathbf{z}$, the target dose function becomes

$$
t\left(\mathbf{x}^{\prime}\right)=\left\{\begin{array}{ll}
0 & \mathbf{x}^{\prime} \in \mathcal{S}_{o} \\
\tau\left(\mathbf{x}^{\prime}\right) & \mathbf{x}^{\prime} \in \mathcal{S}_{t}
\end{array}=t(\mathbf{x}-\mathbf{z})= \begin{cases}0 & \mathbf{x}-\mathbf{z} \in \mathcal{S}_{o} \\
\tau(\mathbf{x}-\mathbf{z}) & \mathbf{x}-\mathbf{z} \in \mathcal{S}_{t}\end{cases}\right.
$$

or equivalently, using equations (4) and (5),

$$
t(\mathbf{x}-\mathbf{z})= \begin{cases}0 & \mathbf{z} \in \mathcal{S}_{o}(\mathbf{x}) \\ \tau(\mathbf{x}-\mathbf{z}) & \mathbf{z} \in \mathcal{S}_{t}(\mathbf{x}) .\end{cases}
$$


Equation (9) can then be written as

$\bar{L}_{R}[\mathbf{x}, y(\cdot)]=\int_{\mathbf{z} \in \mathcal{S}_{t}(\mathbf{x})} L[y(\mathbf{x}), \tau(\mathbf{x}-\mathbf{z})] \mathrm{d} F(\mathbf{z})+L[y(\mathbf{x}), 0] \int_{\mathbf{z} \in \mathcal{S}_{o}(\mathbf{x})} \mathrm{d} F(\mathbf{z})$.

Using equations (13) and (14)

$$
L\left[d\left(\mathbf{x}^{\prime}\right), t\left(\mathbf{x}^{\prime}\right)\right]= \begin{cases}w_{o}\left|t\left(\mathbf{x}^{\prime}\right)-d\left(\mathbf{x}^{\prime}\right)\right|^{b_{o}} & \mathbf{x}^{\prime} \in \mathcal{S}_{o}, \\ w_{t}\left|t\left(\mathbf{x}^{\prime}\right)-d\left(\mathbf{x}^{\prime}\right)\right|^{b_{t}} & \mathbf{x}^{\prime} \in \mathcal{S}_{t},\end{cases}
$$

where $w_{o} \geqslant 0\left(b_{o} \geqslant 1\right)$ and $w_{t} \geqslant 0\left(b_{t} \geqslant 1\right)$ are weights (powers) for OAR and CTV, respectively.

Using equations (1) and (17) and substituting equation (19) into (18) gives

$\bar{L}_{R}[\mathbf{x}, y(\cdot)]=\int_{\mathbf{z} \in \mathcal{S}_{t}(\mathbf{x})} w_{t}|\tau(\mathbf{x}-\mathbf{z})-y(\mathbf{x})|^{b_{t}} \mathrm{~d} F(\mathbf{z})+w_{o} y(\mathbf{x})^{b_{o}} \int_{\mathbf{z} \in \mathcal{S}_{o}(\mathbf{x})} \mathrm{d} F(\mathbf{z})$

We see from equation (20) that when $\mathbf{x}$ is fixed and $b_{o}, b_{t} \geqslant 1, \bar{L}_{R}[\mathbf{x}, y(\cdot)]$ is convex in $y(\mathbf{x})$ (as long as $y(\mathbf{x}) \geqslant 0$ ), so finding the function $y^{*}(\mathbf{x})$ that minimizes $\bar{L}_{R}[\mathbf{x}, y(\mathbf{x})]$ simply involves finding the value of $y(\mathbf{x})$, at every point $\mathbf{x}$, that satisfies $\frac{\mathrm{d}}{\mathrm{d} y} \bar{L}_{R}[\mathbf{x}, y(\mathbf{x})]=0$.

For example, if the loss function is a weighted quadratic (i.e., $b_{o}=b_{t}=2$ ) such as assumed in Shepard et al (1999), Li and Xing (2000), Unkelbach and Oelfke (2004) and Rehbinder et al 2004, it can be readily shown that $y(\mathbf{x})$ satisfies

$$
y^{*}(\mathbf{x})=\frac{w_{t} \int_{\mathbf{z} \in \mathcal{S}_{t}(\mathbf{x})} \tau(\mathbf{x}-\mathbf{z}) \mathrm{d} F(\mathbf{z})}{w_{t} \int_{\mathbf{z} \in \mathcal{S}_{t}(\mathbf{x})} \mathrm{d} F(\mathbf{z})+w_{o} \int_{\mathbf{z} \in \mathcal{S}_{o}(\mathbf{x})} \mathrm{d} F(\mathbf{z})} .
$$

\subsection{Homogeneous CTV}

If the CTV is homogeneous, so that the desired radiation has the same value $\tau\left(x^{\prime}\right)=\tau$ for all points $x^{\prime}$ in the CTV, then equation (20) becomes

$$
\begin{aligned}
\bar{L}_{R}[\mathbf{x}, y(\cdot)] & =w_{t}|\tau-y(\mathbf{x})|^{b_{t}} \int_{\mathbf{z} \in \mathcal{S}_{t}(\mathbf{x})} \mathrm{d} F(\mathbf{z})+w_{o} y(\mathbf{x})^{b_{o}} \int_{\mathbf{z} \in \mathcal{S}_{o}(\mathbf{x})} \mathrm{d} F(\mathbf{z}) \\
& =w_{t} p_{t}(\mathbf{x})|\tau-y(\mathbf{x})|^{b_{t}}+w_{o} p_{o}(\mathbf{x}) y(\mathbf{x})^{b_{o}} .
\end{aligned}
$$

Since in this paper we do not consider multiple fractions, which might involve optimal dose distributions that deliver higher doses at certain regions than the target dose (Unkelbach and Oelfke 2004, Löf et al 1995), equation (22) makes clear that the optimal value of $y(\mathbf{x})$ cannot exceed $\tau$ for any $\mathbf{x}$, so it can be re-written as

$$
\bar{L}_{R}[\mathbf{x}, y(\cdot)]=w_{t} p_{t}(\mathbf{x})(\tau-y(\mathbf{x}))^{b_{t}}+w_{o} p_{o}(\mathbf{x}) y(\mathbf{x})^{b_{o}} .
$$

If $b_{o}>1$ and $b_{t}>1$, for a fixed $\mathbf{x}, \bar{L}_{R}[\mathbf{x}, y(\cdot)]$ is convex and differentiable in $y(\mathbf{x})$ (as long as $y(\mathbf{x}) \geqslant 0)$ and, therefore, is minimized by setting the derivative of the above expression to zero, yielding

$$
b_{t} w_{t} p_{t}(\mathbf{x})(\tau-y(\mathbf{x}))^{b_{t}-1}=b_{o} w_{o} p_{o}(\mathbf{x}) y(\mathbf{x})^{b_{o}-1} .
$$

Letting

$$
\alpha(\mathbf{x}) \equiv \frac{b_{o} w_{o} p_{o}(\mathbf{x})}{b_{t} w_{t} p_{t}(\mathbf{x})},
$$

the optimal dose distribution $y^{*}(\mathbf{x})$ at room location $\mathbf{x}$ is the solution to

$$
\left(\tau-y^{*}(\mathbf{x})\right)^{b_{t}-1}-\alpha(\mathbf{x}) y^{*}(\mathbf{x})^{b_{o}-1}=0 .
$$

We consider four special cases: 
1. If $b_{t}=b_{o}=b>1$, then

$$
y^{*}(\mathbf{x})=\frac{\tau}{1+[\alpha(\mathbf{x})]^{\frac{1}{b-1}}} .
$$

2. If $b_{t}=1$ and $b_{o}>1$, then

$$
y^{*}(\mathbf{x})=\min \left\{\tau, \frac{1}{[\alpha(\mathbf{x})]^{\frac{1}{b_{0}-1}}}\right\} .
$$

3. If $b_{o}=1$ and $b_{t}>1$, then

$$
y^{*}(\mathbf{x})=\max \left\{0, \tau-[\alpha(\mathbf{x})]^{\frac{1}{b_{t}-1}}\right\} .
$$

4. If $b_{t}, b_{o}>1$ and $b_{t}$ is an integer, then equation (24) can be written:

$$
\sum_{i=0}^{b_{t}-1}\left(\begin{array}{c}
b_{t}-1 \\
i
\end{array}\right) \tau^{i}(-y(\mathbf{x}))^{b_{t}-1-i}-\alpha(\mathbf{x}) y(\mathbf{x})^{b_{o}-1}=0,
$$

and $y^{*}(\mathbf{x})$ can be calculated by finding the smallest nonnegative real root of the polynomial on the left-hand side of equation (28).

A comparison of these functions and their dependence on the joint cumulative distribution function $F(\mathbf{z})$ is deferred to the discussion in the next subsection.

3.1.1. Illustrative numerical example. Although the preceding results hold for $3 \mathrm{D}$, for ease of illustration, this section shows numerical examples when motion is restricted to two dimensions: the dose distribution is that of a single intensity-modulated radiation field at the plane normal to the field axis and passing through the isocentre (Bortfeld et al 2002). The distribution of $\mathbf{Z}$ is a two-dimensional normal distribution (using a multi-dimensional normal is a common assumption, see Lujan et al (1999), Lam et al (2005)). Incorporating all uncertainty about the value of the systematic error into the random error allows us, without loss of generality, to set the mean to zero. That is, the probability density function of $\mathbf{Z}$ is

$$
f_{\mathbf{Z}}(\mathbf{z} ; \mathbf{0}, \Sigma)=(2 \pi)^{-1}|\Sigma|^{-\frac{1}{2}} \mathrm{e}^{-\frac{1}{2} \mathbf{z}^{T} \Sigma^{-1} \mathbf{z}},
$$

where $\Sigma$ is the covariance matrix

$$
\Sigma=\left[\begin{array}{cc}
\sigma_{1}^{2} & \sigma_{12} \\
\sigma_{12} & \sigma_{2}^{2}
\end{array}\right]
$$

$\sigma_{i}^{2}=E\left[Z_{i}^{2}\right]$ is the variance of $Z_{i}, i=1,2$, and $\sigma_{12}=\operatorname{Cov}\left(Z_{1}, Z_{2}\right)=E\left[Z_{1} Z_{2}\right]$ (recall that the mean is assumed to be zero) is the covariance of $Z_{1}$ and $Z_{2}$.

Figure 2 represents a simplification of an 'isolated' CTV. Figure 3(a) shows the optimal dose distribution for this simple case when the loss function parameters are $b_{t}=b_{o}=2, w_{t}=$ $15, w_{o}=1, \tau=1$ and, $\Sigma=\left[\begin{array}{ll}1 & 0 \\ 0 & 1\end{array}\right]$. As can be seen, the dose distribution is 'blurred', as will be discussed in section 4.1 .

Figure 4 shows the optimal dose distributions for the special cases given in equations (25)-(28) at the one-dimensional slice (the white line) shown in figure 2 when $w_{t}=15, w_{o}=$ $1, \tau=1$ and, $\Sigma=\left[\begin{array}{ll}1 & 0 \\ 0 & 1\end{array}\right]$. As can be seen, the form of the WPLF, and in particular the values of power parameters $b_{t}$ and $b_{o}$ (see section 4.3), has a significant effect on the shape of the resulting optimal dose distribution.

Furthermore, figure 5 shows the impact of the joint cumulative distribution function of $\mathbf{Z}$ on the optimal dose distribution at the same one-dimensional slice when the parameters of the WPLF are $b_{t}=b_{o}=2, \tau=1, w_{t}=15$, and $w_{o}=1$. In order to make the comparisons 


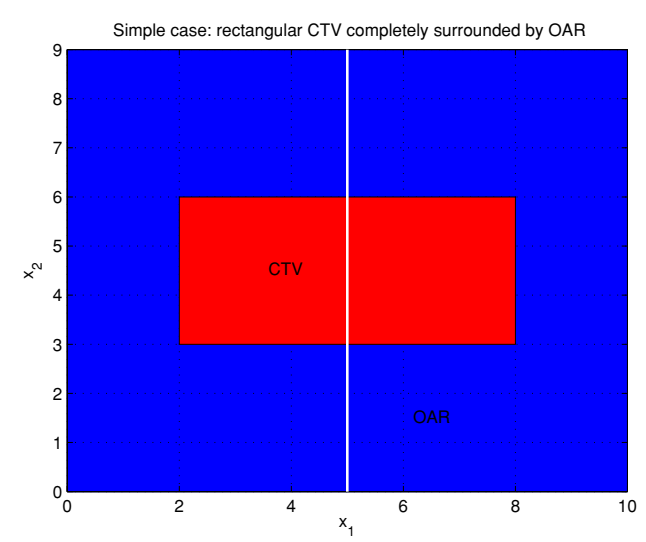

Figure 2. Case I: rectangular CTV completely surrounded by OAR. Patient is in nominal position.

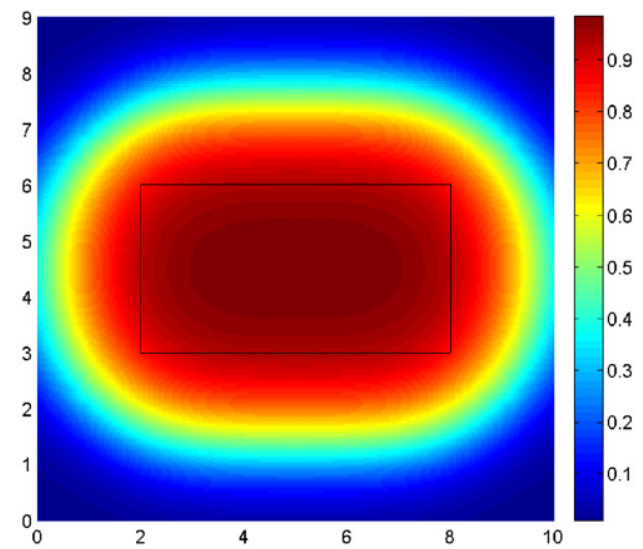

(a)

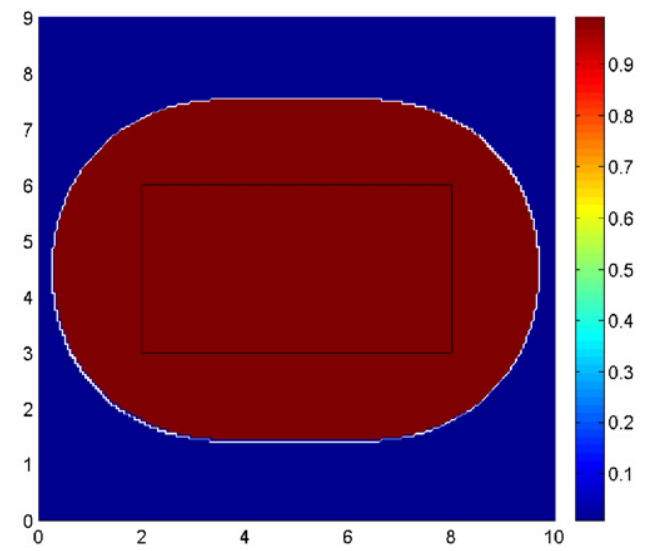

(b)

Figure 3. (a) Optimal blurred dose distribution compared to (b) optimal margin dose distribution for CTV geometry shown in figure 2. $\tau=1, w_{t}=15, w_{o}=1$ and $\Sigma=\left[\begin{array}{ll}1 & 0 \\ 0 & 1\end{array}\right]$, (a) $b_{t}=2, b_{o}=2$ (b) $b_{t}=1, b_{o}=1$.

meaningful, we use joint cumulative distribution functions with equal marginal standard deviations. Note that the optimal dose distribution when the random displacement is uniformly distributed falls off more sharply than when the random displacement is normally distributed, and is zero beyond a certain point. This is not surprising because, unlike the normal distribution, the uniform does not have an infinitely long 'tail'.

Additional numerical examples, exploring the effect of parameter values on the shape of the optimal dose distribution, will be discussed in the following sections.

\subsection{When $b_{t}=b_{o}=1 a$ 'margin' dose distribution is optimal}

In the previous subsection the four special cases required $b_{t}>1$ and/or $b_{o}>1$. A fifth special case, where $b_{t}=b_{o}=1$, is of great interest, but requires a somewhat different analysis. In this case the loss is proportional to the absolute value of the deviation of delivered radiation 


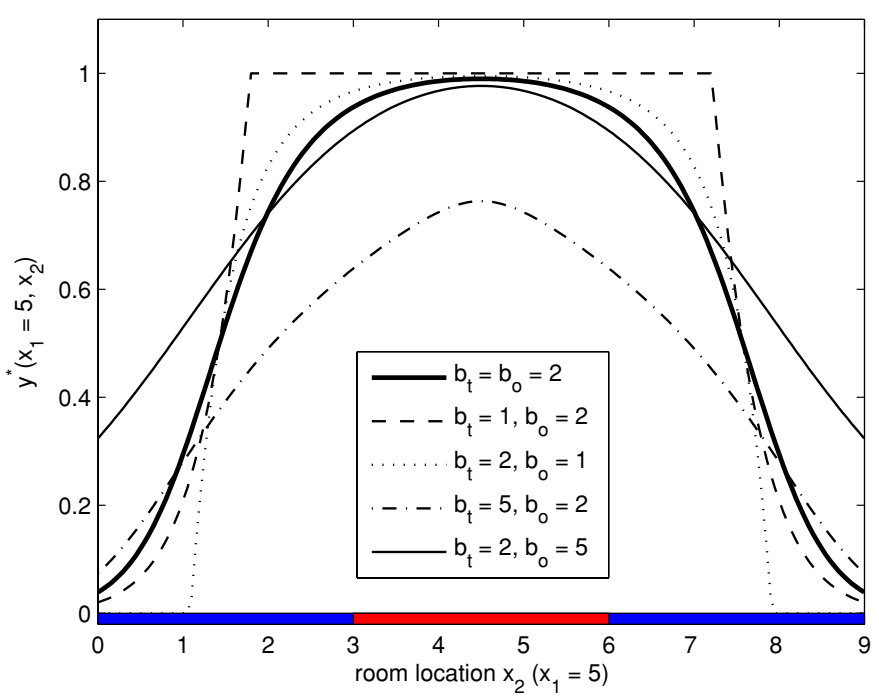

Figure 4. Optimal dose distributions for the special cases given in equations (25)-(28) at the one-dimensional slice (the white line) shown in figure 2. $\tau=1, w_{t}=15, w_{o}=1$ and $\Sigma=\left[\begin{array}{ll}1 & 0 \\ 0 & 1\end{array}\right]$. The nominal position of the CTV is shown in red.

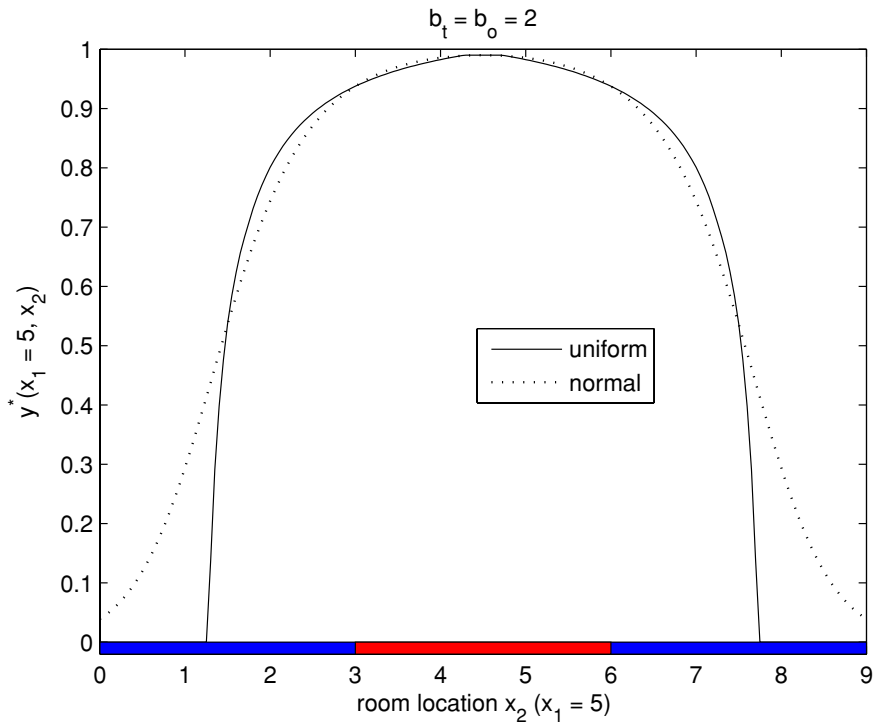

Figure 5. Comparison of optimal dose distributions when $b_{t}=b_{o}=2$ at the one-dimensional slice (the white line) shown in figure 2 for different joint cumulative distribution functions (with the same marginal standard deviations) of random displacement of the patient anatomy. $\tau=1, w_{t}=15, w_{o}=1$. The nominal position of the CTV is shown in red.

from the target, and the expected loss at point $\mathbf{x}$ is

$$
\begin{aligned}
\bar{L}_{R}[\mathbf{x}, y(\cdot)] & =w_{t} p_{t}(\mathbf{x})(\tau-y(\mathbf{x}))+w_{o} p_{o}(\mathbf{x}) y(\mathbf{x}) \\
& =w_{t} p_{t}(\mathbf{x})[\tau-y(\mathbf{x})(1-\alpha(\mathbf{x}))],
\end{aligned}
$$


where $\alpha(\mathbf{x})$ is now given by

$$
\alpha(\mathbf{x}) \equiv \frac{w_{o} p_{o}(\mathbf{x})}{w_{t} p_{t}(\mathbf{x})}
$$

Again, it is straightforward to show that the optimal value of $y(\mathbf{x})$ is between 0 and $\tau$ for all x. Moreover, since expression (31) is linear in $y(\mathbf{x})$, the optimal dose distribution is readily shown to be

$$
y^{*}(\mathbf{x})=\left\{\begin{array}{ll}
0 & \alpha(\mathbf{x})>1, \\
\tau & \alpha(\mathbf{x}) \leqslant 1,
\end{array}= \begin{cases}0 & w_{o} p_{o}(\mathbf{x})>w_{t} p_{t}(\mathbf{x}) \\
\tau & w_{o} p_{o}(\mathbf{x}) \leqslant w_{t} p_{t}(\mathbf{x})\end{cases}\right.
$$

This step function is in fact exactly the PTV created by using a border defined by the set of $\mathbf{x}$ such that $\alpha(\mathbf{x})=1$. That is, the dose distribution calls for no dose at points where $\alpha(\mathbf{x})>1$ and CTV target dose $\tau$ at points where $\alpha(\mathbf{x}) \leqslant 1$.

The resulting expected cost, using equation (31), is

$$
\bar{L}_{R}^{*}(\mathbf{x})=w_{t} p_{t}(\mathbf{x})[\tau-y(\mathbf{x})(1-\alpha(\mathbf{x}))]= \begin{cases}w_{t} p_{t}(\mathbf{x}) \tau & \alpha(\mathbf{x})>1, \\ w_{o} p_{o}(\mathbf{x}) \tau & \alpha(\mathbf{x}) \leqslant 1 .\end{cases}
$$

There are two important features of this result:

- The border defined by the set $\{\mathbf{x}: \alpha(\mathbf{x})=1\}$ is in fact the best margin border (with respect to the WPLF loss function with $b_{t}=b_{o}=1$ ), since it is the result of a cost-minimization procedure;

- It can be shown that no WPLF other than one that is linear in the deviation from target (i.e., with $b_{t}=b_{o}=1$ ) will lead to a cost-optimal dose distribution that has this step function (i.e., 'margin') structure.

Figure 3(b) shows the optimal margin dose distribution for the simple geometry shown in figure 2 with parameters $b_{t}=b_{o}=1, w_{t}=15, w_{o}=1, \tau=1$ and $\Sigma=\left[\begin{array}{ll}1 & 0 \\ 0 & 1\end{array}\right]$. Figure 6 shows the optimal dose distributions with margin structure for different joint cumulative distribution functions. Note that the best margin for the normally distributed random displacement is slightly larger than that for the uniformly distributed random displacement, an effect due to the infinitely long 'tail' of the normal distribution.

\subsection{General case with more than two treatment regions}

The results of sections 3.1 and 3.2 can be generalized for patient anatomies with more than two specified treatment regions (e.g., several CTVs with different target doses, several OARs with different thresholds, and unspecified healthy tissue). Let $\mathcal{S}_{1}, \mathcal{S}_{2}, \ldots, \mathcal{S}_{N}$ be $N$ (threedimensional) regions on the patient. Regions $\mathcal{S}_{1}, \mathcal{S}_{2}, \ldots, \mathcal{S}_{m}$ are CTVs with target dose $\tau_{1}$, $\tau_{2}, \ldots, \tau_{m}$, respectively (each CTV is homogenous), and regions $\mathcal{S}_{m+1}, \mathcal{S}_{m+2}, \ldots, \mathcal{S}_{N}$ have zero target dose, i.e., they are the healthy regions and OARs that should be spared. Equation (22) can be generalized as:

$$
\begin{aligned}
\bar{L}_{R}[\mathbf{x}, y(\cdot)] & =\sum_{i=1}^{m} w_{i}\left|\tau_{i}-y(\mathbf{x})\right|^{b_{i}} \int_{\mathbf{z} \in \mathcal{S}_{i}(\mathbf{x})} \mathrm{d} F(\mathbf{z})+\sum_{j=m+1}^{N} w_{j} y(\mathbf{x})^{b_{j}} \int_{\mathbf{z} \in \mathcal{S}_{j}(\mathbf{x})} \mathrm{d} F(\mathbf{z}) \\
& =\sum_{i=1}^{m} w_{i} p_{i}(\mathbf{x})\left|\tau_{i}-y(\mathbf{x})\right|^{b_{i}}+\sum_{j=m+1}^{N} w_{j} p_{j}(\mathbf{x}) y(\mathbf{x})^{b_{j}},
\end{aligned}
$$

where $w_{i}$ and $b_{i}$ are the weight and power parameters for region $\mathcal{S}_{i}$, respectively, and $p_{i}(\mathbf{x})=\int_{\mathbf{z} \in \mathcal{S}_{i}(\mathbf{x})} \mathrm{d} F(\mathbf{z})$ is the probability that a point at room location $\mathbf{x}$ will be covered by region $\mathcal{S}_{i}$. 


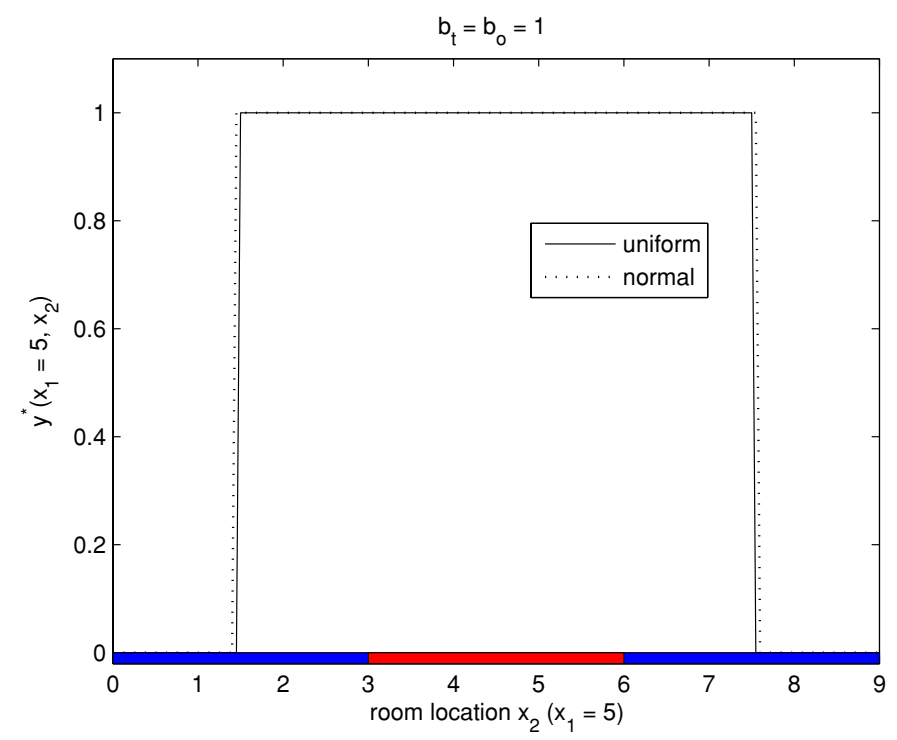

Figure 6. Comparison of optimal dose distributions when $b_{t}=b_{o}=1$ at the one-dimensional slice (the white line) shown in figure 2 for different joint cumulative distribution functions (with the same marginal standard deviations) of random displacement of the patient anatomy. $\tau=1, w_{t}=15, w_{o}=1$. The nominal position of the CTV is shown in red.

If $b_{i}>1, i=1,2, \ldots, N$, for a fixed $\mathbf{x}, L_{R}[\mathbf{x}, y(\cdot)]$ is convex and differentiable in $y(\mathbf{x})$ (as long as $y(\mathbf{x}) \geqslant 0)$. Therefore the optimal dose distribution $y^{*}(\mathbf{x})$ at room location $\mathbf{x}$ is the solution to

$$
\sum_{i=1}^{m} w_{i} p_{i}(\mathbf{x}) b_{i}\left|\tau_{i}-y^{*}(\mathbf{x})\right|^{b_{i}-1} \operatorname{sign}\left(y^{*}(\mathbf{x})-\tau_{i}\right)+\sum_{j=m+1}^{N} w_{j} p_{j}(\mathbf{x}) b_{j} y^{*}(\mathbf{x})^{b_{j}-1}=0,
$$

where

$$
\operatorname{sign}(x)=\left\{\begin{array}{cl}
1, & \text { if } x \geqslant 0 \\
-1, & \text { otherwise }
\end{array}\right.
$$

$y^{*}(\mathbf{x})$ can be calculated using techniques similar to that described in section 3.1.

In a generalization of the special case of section 3.2 , where $b_{i}=1, i=1,2, \ldots, N$, the expected loss at point $\mathbf{x}$ in the room can be written as

$$
\bar{L}_{R}[\mathbf{x}, y(\cdot)]=\sum_{i=1}^{m} w_{i} p_{i}(\mathbf{x})\left|\tau_{i}-y(\mathbf{x})\right|+\sum_{j=m+1}^{N} w_{j} p_{j}(\mathbf{x}) y(\mathbf{x}) .
$$

Equation (37) shows that, for a fixed $\mathbf{x}, L_{R}[\mathbf{x}, y(\cdot)]$ is a piece-wise linear function of $y(\mathbf{x})$, and therefore the optimal dose distribution $y^{*}(\mathbf{x})$ must take one of the values in the set $\left\{0, \tau_{1}, \tau_{2}, \ldots, \tau_{m}\right\}$. In other words, the optimal dose distribution has a step function structure with possibly multiple steps.

\section{Examples for various geometries and treatment loss function parameters}

In this section, we present ideal spatial dose distributions calculated for the three cases of patient geometry with varying complexity, shown in figures 2,8 and 10 . In all of these 


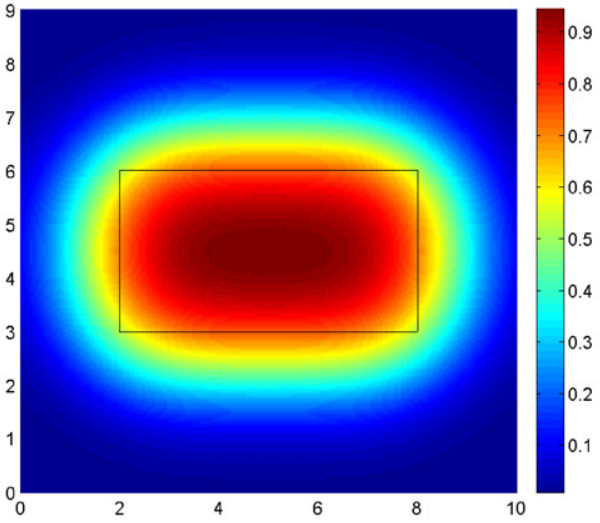

(a)

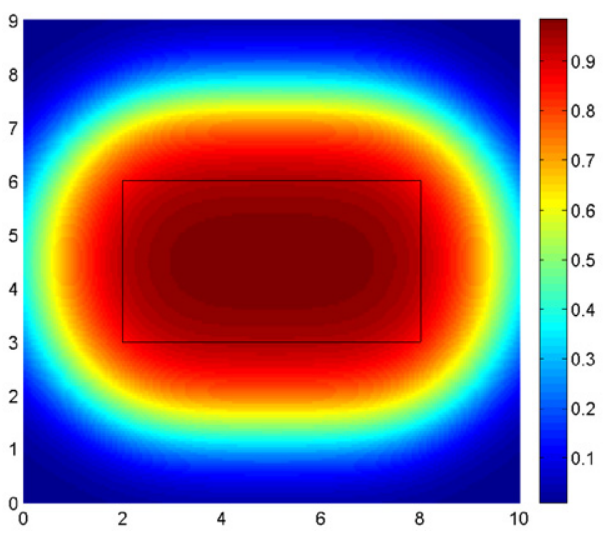

(c)

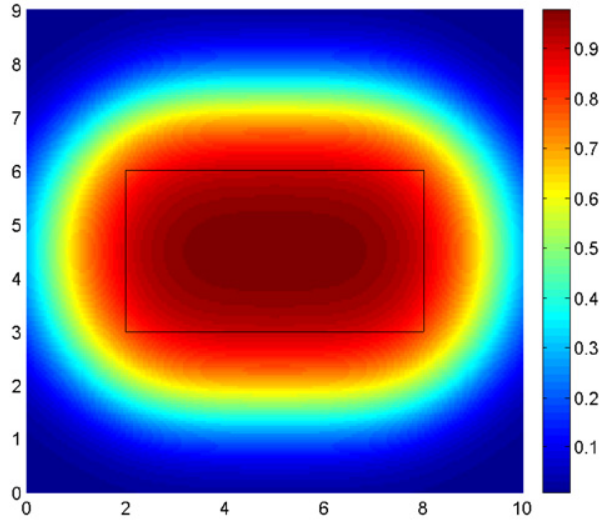

(b)

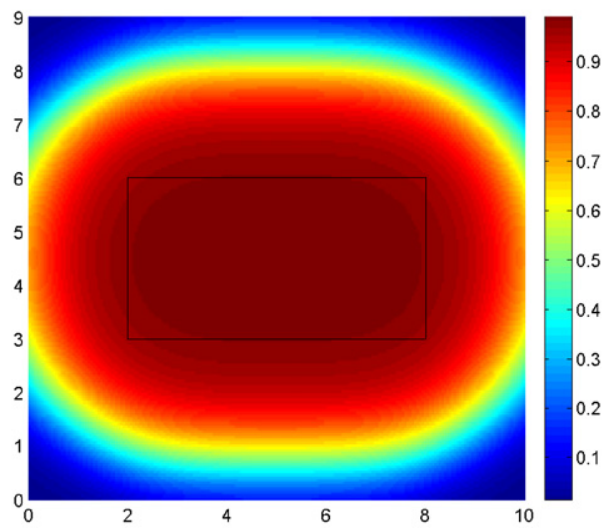

(d)

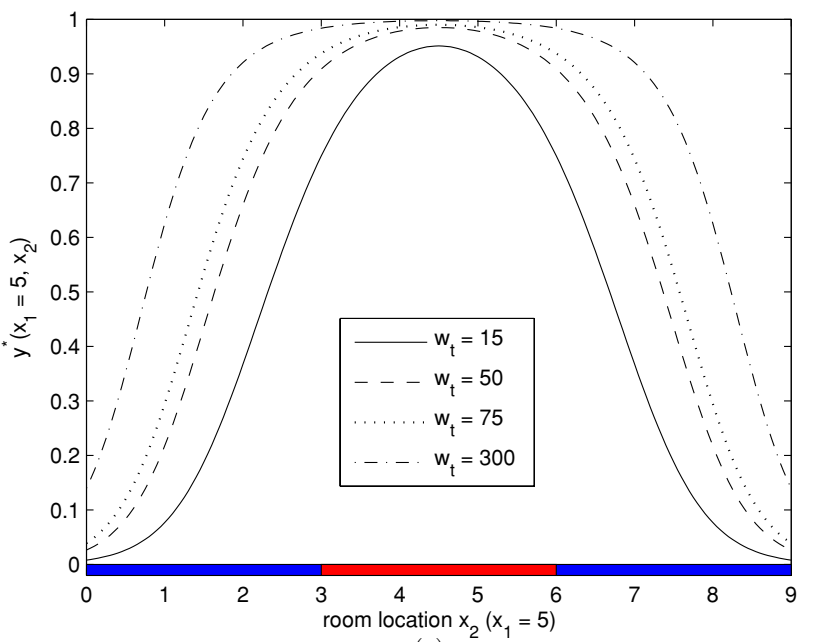

(e)

Figure 7. Effect of weight parameters: optimal dose distributions $y^{*}(\mathbf{x})$ for case I shown in figure 2 for quadratic loss function $\left(b_{t}=b_{o}=2\right)$ and various values of $w_{t}$ with $w_{o}=5, \tau=1$, and zero-mean normal random displacement with $\Sigma=\left[\begin{array}{ll}1 & 0 \\ 0 & 1\end{array}\right]$. (a) $w_{t}=15$, (b) $w_{t}=50$, (c) $w_{t}=75$, (d) $w_{t}=300$, (e) effect of various values of $w_{t}$ at the one-dimensional slice (the white line) shown in figure 2 . The nominal position of the CTV is shown in red. 


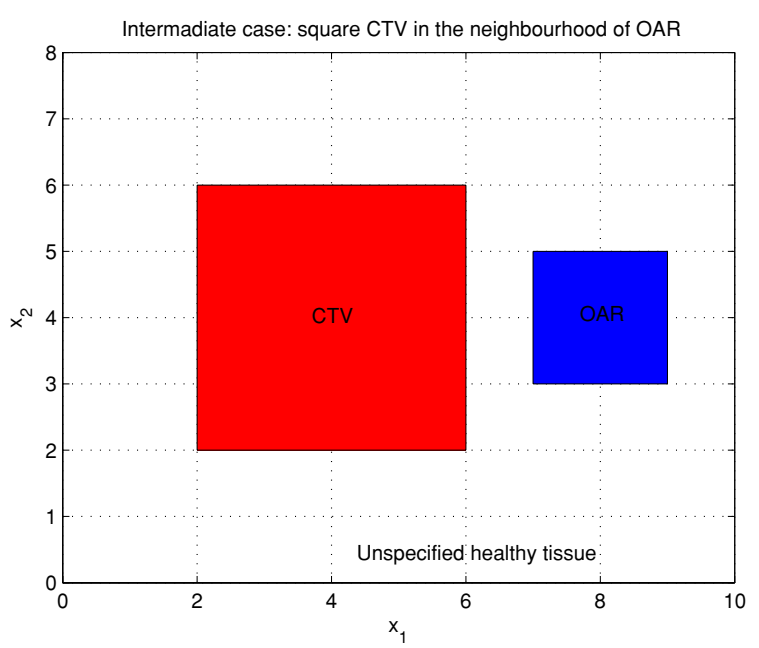

Figure 8. Case II: square CTV in the neighbourhood of an OAR, surrounded by unspecified healthy tissue. Patient is in nominal position.

the patient is in the nominal position. We also analyse the impact of various parameters of the treatment loss function and the displacement distribution on the computed optimal dose distributions.

\subsection{Optimal blurred dose distribution versus optimal 'margin' dose distribution}

Figure 3 shows the optimal dose distribution for the simple CTV geometry shown in figure 2 with $\tau=1, w_{t}=15$, and $w_{o}=1$, and covariance matrix $\Sigma=\left[\begin{array}{ll}1 & 0 \\ 0 & 1\end{array}\right]$ (i.e., random displacements along different axes are uncorrelated). In figure $3(\mathrm{a}), b_{t}=b_{o}=2$, and the optimal dose distribution is 'blurred.' In contrast, when $b_{t}=b_{o}=1$, the optimal dose distribution has a fixed margin, as seen in figure 3(b).

As can be seen in figure 3(a), the optimal blurred dose distribution rises smoothly from $y^{*}(\mathbf{x})=0$ in the parts of the OAR that are far away from the CTV to $y^{*}(\mathbf{x})=1$ in the parts of CTV that are far away from the OAR. As can be seen in figure 3(b), the optimal margin dose distribution, however, is either 0 or 1 , the latter area essentially defining a PTV within which the prescribed target dose $\tau=1$ is planned.

In practice, the size of the margin around a CTV used to produce a PTV is commonly calculated using a 'margin recipe': a formula defined in terms of the standard deviation of the random displacement. These recipes are often ambiguous about the shape of the margin for non-spherical CTVs. In contrast, the margin shown in figure 3(b) is not constant in distance from the boundary of the CTV; in fact, it is smaller near the corners of the CTV. This suggests that when designing a margin, not only the shape of the CTV and the standard deviation of $\mathbf{Z}$, but also the shape of the probability density function of $\mathbf{Z}$ should be considered. This is discussed further in section 4.4.

\subsection{Effect of weight parameters on the optimal dose distribution}

Figures 7(a)-(d) show that when $w_{t}$ (the relative cost of under-treating a point in the CTV) increases, the optimal dose distribution $y^{*}(\mathbf{x})$ delivers more dose to the boundary of the CTV 


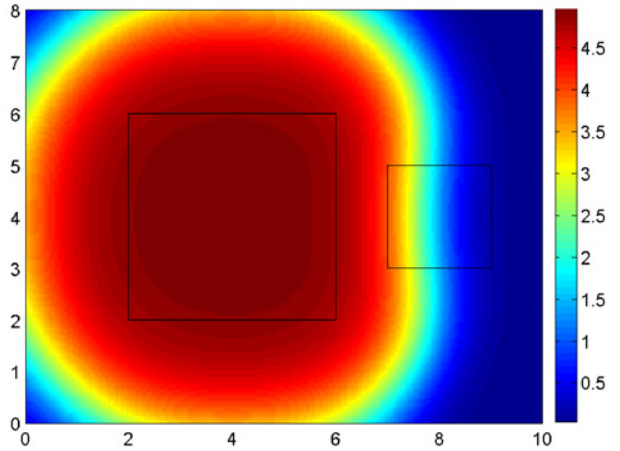

(a)

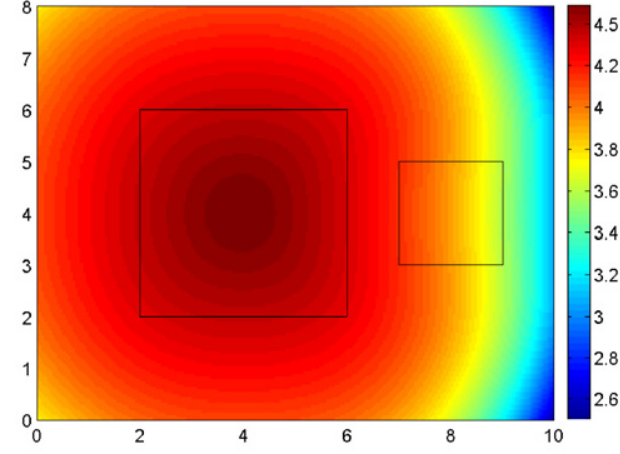

(b)

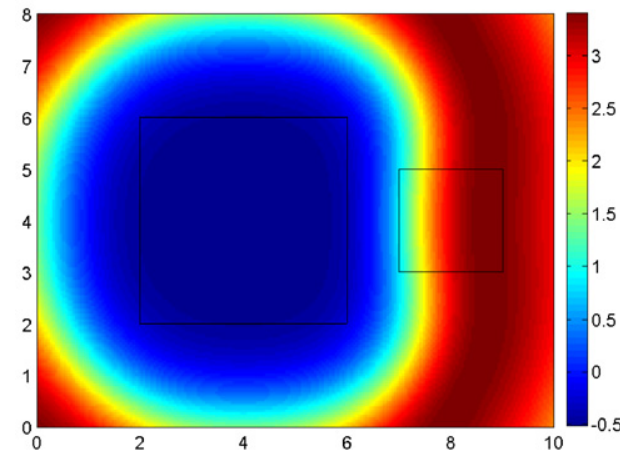

(d)

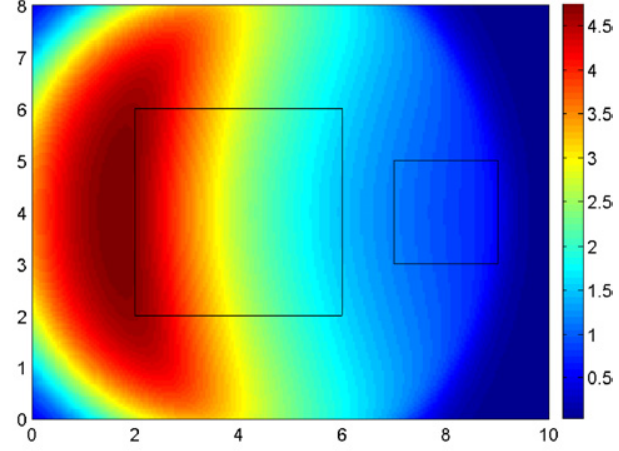

(c)

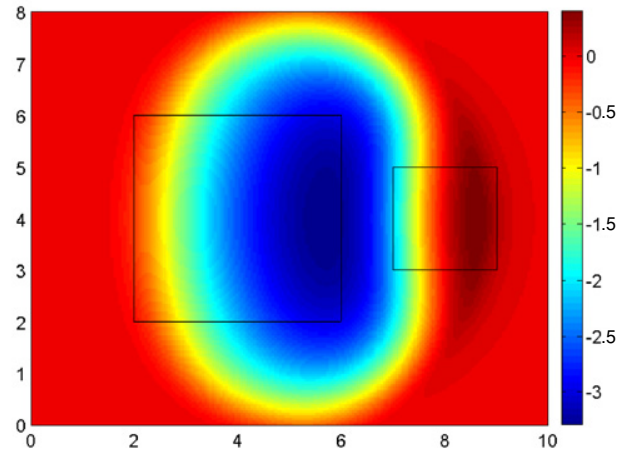

(e)

Figure 9. Effect of power parameters on case II shown in figure 8 with $\tau=5, w_{t}=50, w_{o}=5$, $w_{h}=1$, and $b_{h}=2$, and zero-mean normal random displacement with $\Sigma=\left[\begin{array}{ll}1 & 0 \\ 0 & 1\end{array}\right]$. (a) Reference for comparison: optimal dose distribution for $b_{t}=b_{o}=2$. (b) Optimal dose distribution when $b_{t}=10$ and $b_{o}=2$. (c) Optimal dose distribution when $b_{o}=10$ and $b_{t}=2$. (d) Difference between optimal dose distribution when $b_{t}=10$ in (b) and reference dose distribution in (a). (e) Difference between optimal dose distribution when $b_{o}=10$ in (c) and reference dose distribution in (a).

(and therefore 'covers' the CTV better). The specific effect of $w_{t}$ can be seen more clearly by looking at $y^{*}\left(x_{1}=5, x_{2}\right)$ in figure $7(\mathrm{e})$. As $w_{o}$ (the relative cost of delivering dose to a point in the OAR) increases, it can be shown that it has a similar effect: less dose is delivered to the OAR points. 


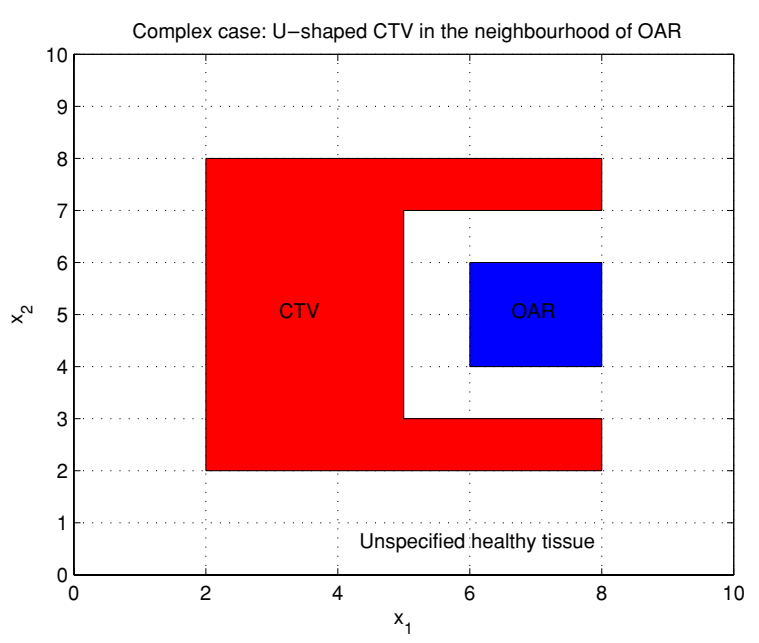

Figure 10. Case III: U-shaped CTV in the neighbourhood of an OAR. Patient is in nominal position.

\subsection{Effect of power parameters on the optimal dose distribution}

Consider case II: the geometry shown in figure 8 and a WPLF with weight parameters $w_{t}, w_{o}$, and $w_{h}$ and power parameters $b_{t}, b_{o}$, and $b_{h}$ for the CTV, OAR, and the unspecified healthy tissue, respectively. These parameters give rise to a loss function that achieves a particular tradeoff between the goal of achieving a prescribed dose, $\tau$, to the CTV versus the goal of sparing the OAR and healthy tissue. It is common practice to raise (lower) the weight parameter of a treatment region in order to emphasize (de-emphasize) the treatment objective for that region. It is also common to change the relative values of $b_{t}, b_{o}$, and $b_{h}$ to achieve the same effect. In section 4.2 it was shown that raising the weight parameter of a treatment region indeed emphasizes the treatment objective of that region. In this section, we assess the impact of power parameters on the dose distribution while keeping the other parameters constant.

Figure 9(a) shows the optimal dose distribution for case II with respect to a WPLF with $b_{t}=b_{o}=2$. Figures 9(b) and (c) show the optimal dose distributions with respect to a WPLF with $b_{t}=10$ and $b_{o}=2$ and a WPLF with $b_{t}=2$ and $b_{o}=10$, respectively. These dose distributions will serve as the reference for comparisons. Figure 9(d) shows the difference between the reference dose distribution in figure 9(a) and the optimal dose distribution $y^{*}(\mathbf{x})$ when $b_{t}=10$ shown in figure 9(b). As can be seen from the figure, the value of the optimal dose distribution at the CTV points when $b_{t}=10$ is smaller than that when $b_{t}=2$. This is contrary to a common intuitive notion that raising the power parameter $b_{t}$ results in a higher dose to the CTV.

Figure 9(e) shows the difference between the reference dose distribution in figure 9(a) and the optimal dose distribution when $b_{o}=10$ shown in figure $9(\mathrm{c})$. Although points in the OAR which are closer to the CTV receive lower doses when the power parameter $b_{o}$ is raised from 2 to 10, points further away from the CTV receive higher doses.

To explain the above, perhaps counterintuitive, observations, consider the structure of the expected loss function $\bar{L}_{R}[\mathbf{x}, y(\cdot)]$ at room location $\mathbf{x}$, for the case II geometry shown in figure 8 (see equation (35)):

$$
\bar{L}_{R}[\mathbf{x}, y(\cdot)]=w_{t} p_{t}(\mathbf{x})|\tau-y(\mathbf{x})|^{b_{t}}+w_{o} p_{o}(x) y(\mathbf{x})^{b_{o}}+w_{h} p_{h}(x) y(\mathbf{x})^{b_{h}} .
$$


Recall that $p_{t}(\mathbf{x}), p_{o}(\mathbf{x})$ and $p_{h}(\mathbf{x})$ are the probabilities that a point at the room location $\mathbf{x}$ will be covered by the CTV, OAR and healthy tissue regions, respectively.

Since the optimal value of $y(\mathbf{x})$ should never exceed $\tau$ for any $\mathbf{x}$, equation (38) can be re-written as

$$
\begin{aligned}
\bar{L}_{R}[\mathbf{x}, y(\cdot)] & =w_{t} p_{t}(\mathbf{x})(\tau-y(\mathbf{x}))^{b_{t}}+w_{o} p_{o}(x) y(\mathbf{x})^{b_{o}}+w_{h} p_{h}(x) y(\mathbf{x})^{b_{h}} \\
& =w_{t} p_{t}(\mathbf{x}) \tau^{b_{t}}(1-\widehat{y}(\mathbf{x}))^{b_{t}}+w_{o} p_{o}(x) \tau^{b_{o}} \widehat{y}(\mathbf{x})^{b_{o}}+w_{h} p_{h}(x) \tau^{b_{h}} \widehat{y}(\mathbf{x})^{b_{h}},
\end{aligned}
$$

where $\widehat{y}(\mathbf{x}) \equiv y(\mathbf{x}) / \tau$. Obviously, $0 \leqslant \widehat{y}(\mathbf{x}) \leqslant 1$ and $0 \leqslant 1-\widehat{y}(\mathbf{x}) \leqslant 1$. Therefore, when $b_{t}$ is raised, $\tau^{b_{t}}$ increases, while $(1-\widehat{y}(\mathbf{x}))^{b_{t}}$ decreases. Similarly, when $b_{o}$ is raised, $\tau^{b_{o}}$ increases, while $\widehat{y}(\mathbf{x})^{b_{o}}$ decreases. Which of these terms dominates when the power parameters are raised depends on the relationship between the values of $\tau, b_{t}, b_{o}, b_{h}$, and $\hat{y}(x)$, as demonstrated by the numerical results. As can be seen in figures $9(\mathrm{~d})$ and (e), raising the power parameter of the treatment region can decrease the optimal value of $y(\cdot)$ at some points, and raise it at other points of the same treatment region.

In more complicated (and more realistic) instances of the planning problem, where multiple CTVs with various target doses may be present (see the derivation in section 3.3), and where components of a power loss function may have more sophisticated definitions (e.g., Kessler et al (2005)), these counter-intuitive effects may not be as pronounced. However, this simple analysis demonstrates that the fundamental properties of the loss function, and the resulting optimal dose distribution, need to be carefully considered in the design of radiation treatments. The examples serve to underscore the impact of these properties, and our analysis provides tools that can be generalized and utilized for realistic planning problems in the future.

\subsection{Effect of covariance matrix $\Sigma$ on the optimal dose distribution}

Figure 11 shows various optimal 'margin' dose distributions $\left(b_{t}=b_{o}=b_{h}=1\right)$ and optimal 'blurred' dose distributions $\left(b_{t}=b_{o}=b_{h}=2\right)$, for the case III geometry shown in figure 10 , with $\tau=1, w_{t}=50, w_{o}=5, w_{h}=1$, and various covariance matrices $\Sigma$. As can be seen in these figures, the shape of the probability density function of $\mathbf{Z}$, which is determined by the covariance matrix $\Sigma$, has a great influence on the shape of the optimal dose distributions. Therefore, if measurement data indicate that there is correlation between random displacement along different axes of the coordinate system used in treatment planning, it should be incorporated into the treatment plan.

\section{Optimal deliverable dose distributions}

In previous sections, we focused on calculating ideal spatial dose distributions. Unfortunately, delivering these may be difficult in practice. A more realistic approach might be to minimize the total expected loss given in equation (10) with respect to the beamlet intensities. However, this problem presents a much more difficult mathematical and computational challenge, requiring additional simplifications and assumptions to make it tractable.

On the other hand, one could use the ideal spatial dose distributions as a target dose for beamlet optimization. This is a mathematically simpler problem than minimizing the total expected loss.

Although these two problems are not equivalent, preliminary computations show that, under reasonable assumptions about the shape of the dose deposition kernel and the joint cumulative distribution function of the random displacement, the deliverable optimal dose distributions and associated fluence maps that result from the two approaches are very close to each other. 


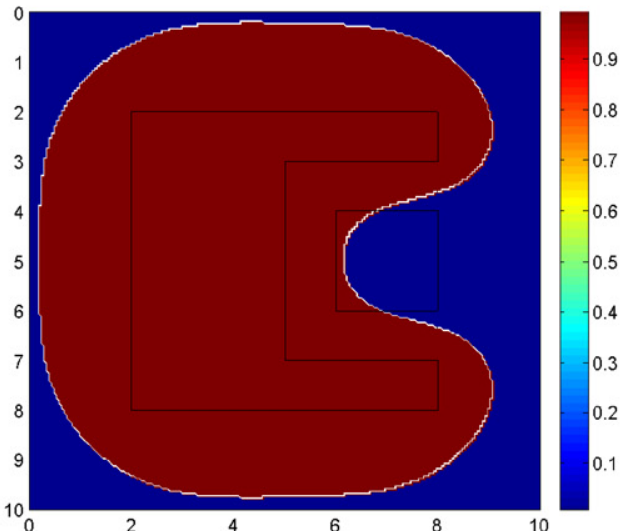

(a)

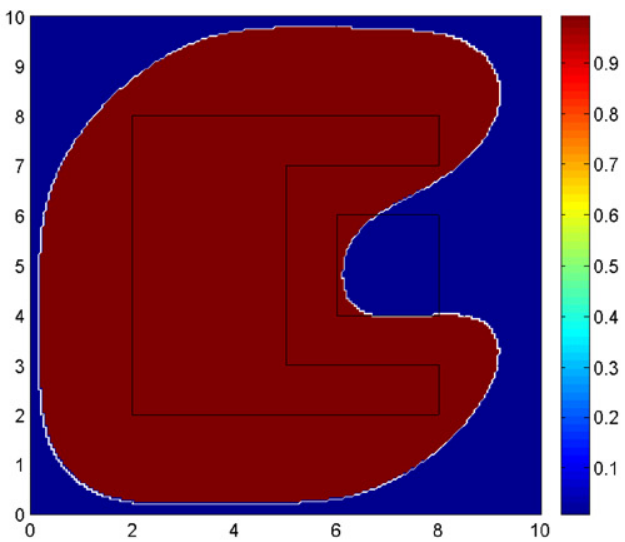

(c)

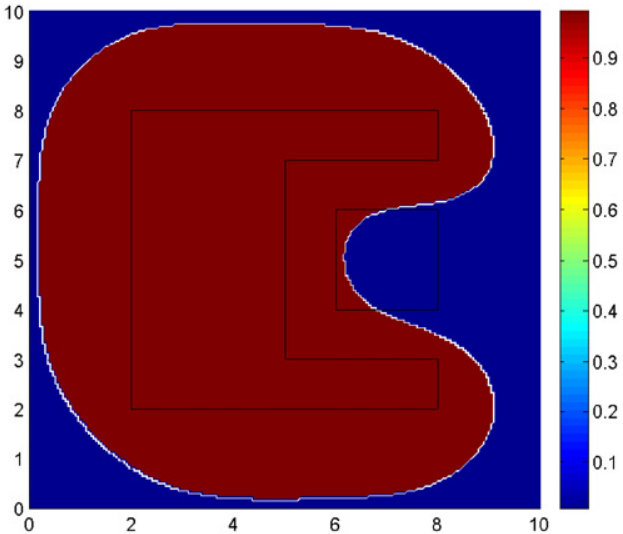

(e)

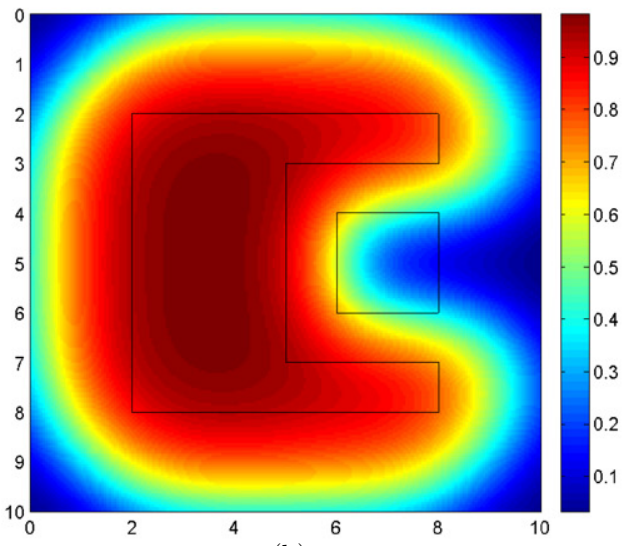

(b)

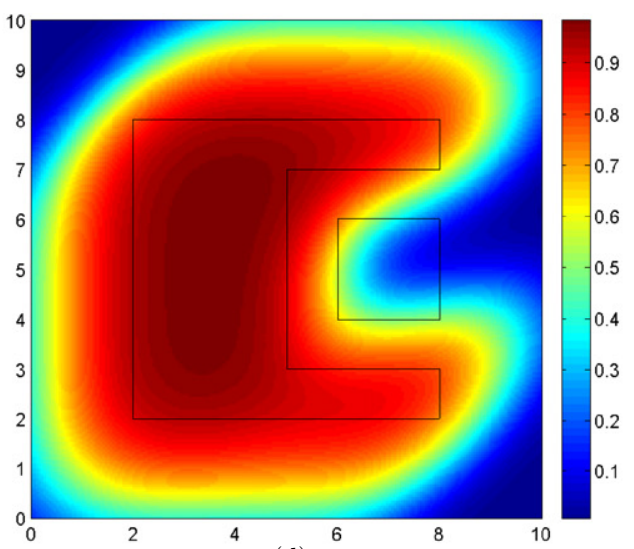

(d)

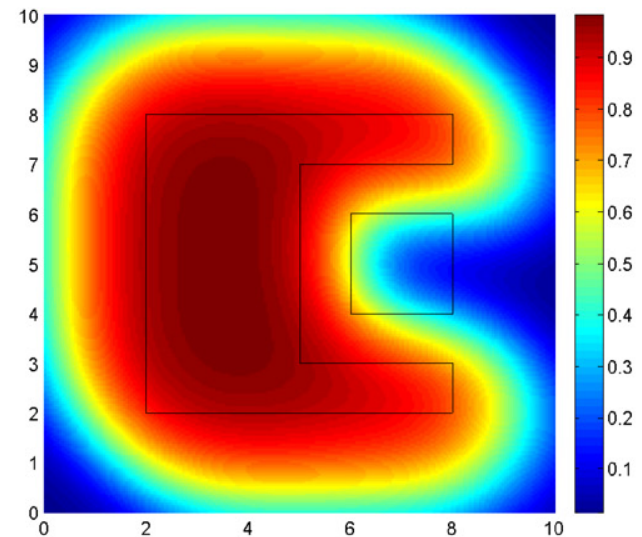

(f)

Figure 11. Effect of covariance matrix $\Sigma$ : optimal dose distributions $y^{*}(\mathbf{x})$ for case III geometry shown in figure 10 with $\tau=1, w_{t}=50, w_{o}=5, w_{h}=1$ and zero-mean normal random displacement with $\Sigma=\left[\begin{array}{cc}1 & \sigma_{12} \\ \sigma_{12} & 1\end{array}\right]$, where $\sigma_{12}$, covariance of $Z_{1}$ and $Z_{2}$, is varied. (a) $\sigma_{12}=0$, $b_{T}=b_{o}=b_{h}=1$, (b) $\sigma_{12}=0, b_{T}=b_{o}=b_{h}=2$, (c) $\sigma_{12}=0.5, b_{T}=b_{o}=b_{h}=1$, (d) $\sigma_{12}=$ $0.5, b_{T}=b_{o}=b_{h}=2$, (e) $\sigma_{12}=-0.2, b_{T}=b_{o}=b_{h}=1$, (f) $\sigma_{12}=-0.2, b_{T}=b_{o}=b_{h}=2$. 


\section{Summary and conclusions}

Optimal (i.e., expected cost minimizing) dose distributions — in general — do not exhibit sharp margins: the dose is essentially zero over the OAR and healthy regions that are far away from the CTV, rise smoothly over the regions that are in the vicinity of the boundary between the OAR or healthy tissue and CTV, and are close to the prescribed target dose over regions of the CTV that are far away from the OAR and healthy tissue.

No WPLF other than one that is proportional to a linear deviation from target (i.e., WPLF with $b_{i}=1, i=1,2, \ldots, N$ where $N$ is the number of treatment regions in the patient's anatomy) will lead to a cost-optimal dose distribution that has a margin structure. As a consequence, although margins may be easier to think about, they are not optimal unless the underlying measure of performance (or loss function) in evaluating the dose distribution is a WPLF with $b_{i}=1, i=1,2, \ldots, N$.

Analysis and numerical experiments show that the common practice of raising powers to penalize deviation from target may be counter-productive.

Moreover, the shape of the probability distribution of the random displacement can have a significant effect on the shape of optimal blurred and optimal margin dose distributions, and therefore, correlation of motion along different axes must be taken into account in the design of dose distributions.

Finally, we re-emphasize the fact that, although our results offer insights into desirable dose distributions, actually delivering them may be difficult, if not impossible. However, there is an extensive literature on the development of fluence patterns, commonly known as inverse planning, to deliver a desired dose distribution, or one which is as close to the desired dose distribution as possible. Our results can be used in conjunction with such well-established inverse planning procedures to produce radiotherapy dose distributions that are robust against random displacements.

\section{Acknowledgments}

This work was supported in part by National Institute of Health (NIH) Grant P01CA59872 and by National Science Foundation (NFS) Grant CCF-0306240.

\section{References}

Alber M and Nüsslin F 1999 An objective function for radiation treatment optimization based on local biological measures Phys. Med. Biol. 44 479-93

Baum C, Alber M, Birkner M and Rietzel E 2006 Robust treatment planning for intensity modulated radiotherapy of prostate cancer based on coverage probabilities Radiother. Oncol. 78 27-35

Bortfeld T, Jiang S B and Rietzel E 2004 Effects of motion on the total dose distribution Semin. Radiat. Oncol. 14 41-51

Bortfeld T, Jokivarsi K, Goitein M, Kung J and Jiang S B 2002 Effects of intra-fraction motion on IMRT dose delivery: statistical analysis and simulation Phys. Med. Biol. 47 2203-20

ICRU 1993 Prescribing, recording and reporting photon beam therapy ICRU Report 50 (Bethesda, MD: International Commission on Radiation Units and Measurements)

Kessler M L, Mcshan D L, Epelman M A, Vineberg K A, Eisbruch A, Lawrence T S and Fraass B A 2005 Costlets: a generalized approach to cost functions for automated optimization of IMRT treatment plans Optim. Eng. $6421-48$

Killoran J H, Kooy H M, Gladstone D J, Welte F J and Beard CJ 1997 A numerical simulation of organ motion and daily setup uncertainties: implications for radiation therapy Int. J. Radiat. Oncol. Biol. Phys. 37 $213-21$

Lam K L, Ten Haken R K, Litzenberg D, Balter J M and Pollock S M 2005 An application of Bayesian statistical methods to adaptive radiotherapy Phys. Med. Biol. $503849-58$

Li J G and Xing L 2000 Inverse planning incorporating organ motion Med. Phys. 27 1573-8 
Löf J, Lind B K and Brahme A 1995 Optimal radiation beam profiles considering the stochastic process of patient positioning in fractionated radiation therapy Inverse Problems 11 1189-209

Lujan A E, Ten Haken R K, Larsen E W and Balter J M 1999 Quantization of setup uncertainties in 3-D dose calculations Med. Phys. 26 2397-402

McKenzie A L, van Herk M and Mijnheer B 2000 The width of margins in radiotherapy treatment plans Phys. Med. Biol. 45 3331-42

Rehbinder H, Forsgren C and Löf J 2004 Adaptive radiation therapy for compensation of errors in patient setup and treatment delivery Med. Phys. 31 3363-71

Shepard D M, Ferris M C, Olivera G H and Mackie T R 1999 Optimizing the delivery of radiation therapy to cancer patients SIAM Rev. $\mathbf{4 1} 721-44$

Stroom J C, De Boer H C, Huizenga H and Visser A G 1999 Inclusion of geometrical uncertainties in radiotherapy treatment planning by means of coverage probability Int. J. Radiat. Oncol. Biol. Phys. 43 905-19

Tsien C, Eisbruch A, McShan D, Kessler M, Marsh R and Fraass B 2003 IMRT for locally advanced paranasal sinus tumour: incorporating clinical decisions in the optimization process Int. J. Radiat. Oncol. Biol. Phys. 55 776-84

Unkelbach J and Oelfke U 2004 Inclusion of organ movements in IMRT treatment planning via inverse planning based on probability distributions Phys. Med. Biol. 49 4005-29

van Herk M 2004 Errors and margins in radiotherapy Semin. Radiat. Oncol. 14 52-64

van Herk M, Remeijer P, Rasch C and Lebesque J V 2000 The probability of correct target dosage: dose-population histograms for deriving treatment margins in radiotherapy Int. J. Radiat. Oncol. Biol. Phys. 47 1121-35 